\title{
Caracterização cinesiológica do choku-tsuki executado com impacto e sua comparação com a execução sem impacto
}

\author{
António M. VENCESBRITO* \\ IPS - Escola Superior de Desporto de Rio Maior (Portugal)
}

Recepción: 16/01/2012; Aceptación: 24/02/2012; Publicación: 15/06/2012.

\begin{abstract}
Resumo
Objectivos: Este trabalho tem como objetivo efetuar a caracterização cinemática e electromiográfica de um soco básico de karaté (choku-zuki) realizado com impacto num alvo (makiwara) por karatecas experientes, e comparar com a execução sem impacto efetivo no alvo (com controlo). Esta caracterização permite ao treinador (sensei) saber como o treino influencia a realização do soco em cada uma destas vertentes, e a partir dessa informação definir estratégias de treino que considere adequadas aos seus objetivos.

Método: Numa amostra de dez karatecas masculinos da seleção Portuguesa foram recolhidos dados cinemáticos e electromiográficos durante a execução do choku-zuki, com impacto e sem impacto, relativamente aos segmentos braço e antebraço.

Resultados: Verificou-se que a actividade cinemática e neuromuscular na realização do choku-zuki acontece num intervalo de tempo inferior a $300 \mathrm{~ms}$. A actividade muscular e segmentar estudada mostra uma tendência de activação sequencial com um sentido próximo-distal, com a musculatura interveniente no segmento braço a ter maior intensidade de ativação que os músculos intervenientes no segmento antebraço. Os movimentos de flexão do braço realizados na articulação do ombro e de extensão do antebraço realizado na articulação do cotovelo são efetuados com grande amplitude. Já a pronação do antebraço realizada na articulação do cotovelo não é completa e é tendencialmente o movimento segmentar que inicia a execução do choku-zuki.

Conclusão: Comparativamente a execução do choku-zuki com impacto e sem impacto é cinesiológicamente diferente. Esta diferença realça a importância que os treinadores deverão dar ao ensino da execução e treino do soco com impacto efetivo no alvo.
\end{abstract}

Palavras-chave: Desportos de Combate, Karaté, Controlo Motor, Cinesiologia, Electromiografia.

\section{Kinesiological characterization of choku-tsuki done with impact and its comparison with its execution without impact}

\begin{abstract}
Aim: This work aims to characterize the kinematics and electromyography of a basic karate punch (choku-zuki) made with an impact on a target (makiwara) by experienced karateka, and compares it with the execution without real impact on the target (control). This characterization allows to the coach (sensei) to know how training influences the performance of the punch in each one of these circumstances, and from that information it is possible to define strategies of training that considers adapted to its goals.

Method: In a sample of ten male karateka of the Portuguese Karaté Team the kinematic and electromyographic data of the arm and forearm segments were collected during the execution of choku-zuki, with impact and no impact.
\end{abstract}

Results: It was found that the kinematics and

\section{Caracterización cinesiológica del choku-tsuki realizado con impacto y comparación con su ejecución sin impacto}

\section{Resumen}

Objetivo: Este trabajo pretende caracterizar la cinemática y electromiografía de un puñetazo básico de kárate (chokuzuki) realizado con impacto sobre un objetivo (makiwara) por karatekas experimentados, y compararlo con la ejecución sin impacto real (control). Esta caracterización permite al entrenador (sensei) conocer cómo influye el entrenamiento en la ejecución del puñetazo en cada una de estas circunstancias, y a partir de esa información es posible definir estrategias de entrenamiento adaptadas a sus objetivos.

Método: En una muestra de diez karatekas de la selección portuguesa se recogieron datos cinemáticos y electromiográficos durante la ejecución del choku-zuki, con impacto y sin impacto, en relación a los segmentos del brazo y del antebrazo.

Resultados: Se verificó que la actividad cinemática y

*E-mail: abrito@esdrm.ipsantarem.pt. Dirección: Escola Superior de Desporto de Rio Maior. Av. Dr. Mário Soares. 2040326. Rio Maior (Portugal). 
neuromuscular activity in the performance of choku-zuki happens in a time interval less than $300 \mathrm{~ms}$. The studied muscles activity and segments of the upper limb shows a trend of activation sequence with a proximal to distal direction, with the intervening segment arm muscles having a greater intensity of activation that the muscles involved in the forearm segment. The arm flexion at the shoulder joint and the forearm extension at the elbow joint are done with greater amplitude, and the forearm pronation is not complete at the time of impact. The forearm pronation at the elbow joint is not complete and tends to be the first segmental movement in the choku-zuki execution.

Conclusion: The executions of choku-zuki with impact and no impact are kinesiologically different. This difference highlights the importance that coaches should provide to teach and training the punch execution with an effective impact on the target.

Keywords: Combat Sports, Karaté, Motor Control, Kinesiology, Electromyography. neuromuscular en la realización del choku-zuki sucede en un intervalo de tiempo inferior a $300 \mathrm{~ms}$. La actividad muscular y segmentaria estudiada muestra una tendencia de activación secuencial, en un sentido próximo-distal, teniendo la musculatura implicada del brazo mayor intensidad de activación que los músculos del antebrazo. Los movimientos de flexión del brazo realizados en la articulación del hombro y de extensión del antebrazo realizados en la articulación del codo son efectuados con gran amplitud. La pronación del antebrazo en la articulación del codo no es completa y tiende a ser el movimiento segmentario que inicia la ejecución del chokuzuki.

Conclusión: Las ejecuciones del choku-zuki con y sin impacto son cinesiológicamente diferente. Esta diferencia realza la importancia que los entrenadores deberían dar a la enseñanza de la ejecución y al entrenamiento del puñetazo con impacto efectivo en el objetivo.

Palabras clave: Deportes de combate, Kárate, Control Motor, Kinesiología, Electromiografía.

\section{1.- Introdução}

No processo de formação e de iniciação do karaté o primeiro soco e o mais simples de aprender é o direto, que descreve uma trajetória retilínea, dirigido à região média do tórax do adversário e executado a partir de uma posição estática (heiko-dachi). Esta técnica é conhecida como choku-zuki e a partir dela os karatecas aprendem formas mais complexas de socar (oi-zuki ou gyaku-zuki). Esta aprendizagem efectua-se a partir de movimentos realizados inicialmente de forma lenta e controlada passando para movimentos mais rápidos e potentes, sem alvo definido e com um alvo. Esta é uma habilidade fundamental no processo de formação do karateka, em que a sua correta aprendizagem permite progressivos ganhos de eficácia e eficiência de execução, e conduz à prevenção de lesões nas estruturas anatómicas do membro superior que derivam dos impactos, que é especialmente importante nas fases de desenvolvimento de indivíduos jovens.

o choku-zuki é um exemplo de uma acção balísticos ao tentar alcançar um alvo o mais rápido possível, onde a curta duração do movimento pode impor sérias limitações propriocetivas e visuais à correcção da sua execução. Esses movimentos são programados centralmente e realizados de acordo com padrões gerados de coordenação neuromuscular relacionados com o objectivo da tarefa (Hallett et al. , 1975; Sanes \& Jennings, 1984). A execução do choku-zuki realiza-se segundo uma acção conjugada e sequencial de participação da cintura pélvica, tronco e membro superior o que permite uma utilização de energia que flui desde a cintura pélvica até ao punho.

Nesta cadeia cinética será relevante a intervenção dos músculos bíceps brachii, triceps brachii, deltoid, teres major, serratus anterior, latissimus dorsi, pectoralis major, external obliqus e do rectus abdominis (Nakayama, 1983; Link \& Chou, 2011). Já Courtonne (1996), ao descrever o chokuzuki isola a acção do membro superior relativamente à intervenção do tronco. Este autor descreve a ação motora a decorrer num percurso de 5 fases, com uma sequência semelhante à referida por Nakayama (1983), mas introduzindo a existência de movimentos de rotação interna e de abdução do braço, em complementaridade da flexão do braço realizados na articulação do ombro, e antecedendo o movimento de pronação do antebraço na articulação do cotovelo. Por outro lado salienta a intervenção dos músculos pronadores e supinadores no antebraço e negligencia a intervenção do músculo triceps brachii.

Estas abordagens de carácter empírico tornam perceptível a existência de um padrão motor pré-definido com uma sequência temporal de desenvolvimento dos movimentos em cada um dos componentes segmentares, com a associação de músculos que vão desenvolver papéis de agonismo e antagonismo. Padrão empírico este que tem sido utlizado pelos treinadores de karaté no ensino desta técnica, com tranfer para os restantes socos. 
Por outro lado surge a referência ao papel do treino como instrumento que permite a alteração do comportamento motor e do rendimento desportivo (performance), melhorando-o, o que vulgarmente se traduz no maior êxito da prestação motora devido a uma maior eficácia na realização do gesto (Schimdt, 1993).

Schimdt e Wrisberg (2000) referem a aprendizagem motora como o resultado de alterações nos processos internos que determinam uma capacidade individual para produzir uma ação motora, em que o nível de aprendizagem motora individual melhora com a prática. Assim, podemos entender o treino sistemático de karaté como um veículo de aprendizagem de tarefas motoras específicas onde se pretende obter, melhorar e automatizar gestos motores em resultado da repetição consciente, tal como Grosser et al. (1986) preconizam para as habilidades motoras. Este treino será então responsável por um comportamento cinesiológico dos karatecas mais adequado á realização da acção motora e à concretização do seu objectivo em tarefas de maior complexidade relativamente às menos complexas.

Através da identificação de padrões cinemáticos e electromiográficos (EMG) em atletas experientes pode caracterizar-se as técnicas motoras, assim como os padrões de coordenação neuromuscular implementados e desenvolvidos através do processo de treino. Esta caracterização pode ser um factor importante para o treinador de karatê, permitindo-lhe definir estratégias de ensino que conduzam a um melhor desempenho, ou evitar erros de aprendizagem e prevenir possíveis lesões que ocorrem com alguma frequência nas extremidades superiores (Zetaruk et al. , 2005; Bledsoe et al. , 2006; Zetaruk et al. , 2000).

O objetivo deste estudo é i) caracterizar os padrões cinemáticos e EMG do soco choku-zuki realizado com impacto por karatecas experientes, e ii) comparar os padrões cinemáticos e EMG do soco choku-zuki realizado por karatecas experientes entre a sua execução com impacto e a execução sem impacto efectivo. A sua concretização fundamentou-se na hipótese de que os padrões cinemáticos e EMG são diferentes entre as duas formas de execução do choku-zuki.

A caracterização da execução do choku-zuki por atletas de alto rendimento permitirá ao treinador (sensei) ter um indicador do resultado do treino efetuado ao longo dos anos em atletas de referência. A partir desse conhecimento poderá adequar o seu processo de ensino/treino e as metodologias utilizadas para aumentar o êxito das aprendizagens e da execução da técnica de socar.

Para a concretização do objectivo proposto, recorremos à análise cinemática tridimensional e à análise electromiográfica do gesto motor, realizado sem constrangimentos limitadores que o tornariam diferente da sua realidade de execução em situação real.

\section{2.- Metodologia}

\section{1.- Amostra}

Participaram neste estudo um grupo de dez atletas karatecas masculinos, pertencentes à seleção portuguesa de karaté, com graduação de Dan, com um tempo médio de prática de $15 \pm 4$ anos, e com mais de quatro anos de experiência competitiva. Todos os participantes eram destros e deram o seu consentimento escrito para a participação no estudo. Na tabela 1 encontrase a caracterização física dos participantes.

Tabela 1

Caracterização física dos participantes

\begin{tabular}{lc}
\hline & Karatecas \\
\hline Idade (anos) & $25 \pm 3$ \\
Altura (cm) & $174.9 \pm 6$ \\
Peso (kg) & $71 \pm 9$ \\
Comprimento membro & $78.4 \pm 5$ \\
superior (cm) & $14 \pm 7.5$ \\
Massa gorda (\%) &
\end{tabular}

\section{2.- Desenho Experimental}

O estudo centrou-se na análise cinesiológica de um movimento de socar do karaté (chokuzuki) num alvo fixo (makiwara). A makiwara consiste numa prancha de madeira com $1.5 \mathrm{~cm}$ de espessura e $134 \mathrm{~cm}$ de comprimento, colocada na vertical e fixa no solo, com a zona alvo a uma altura de $116 \mathrm{~cm}$ do solo. 
A execução do soco foi realizada a partir de um posicionamento inicial dos participantes em pé (heiko-dachi), de frente para a makiwara, distanciados desta pelo comprimento do seu membro superior. Inicialmente $\mathrm{o}$ membro superior direito estava posicionado com o braço com uma extensão no ombro inferior a $40^{\circ}$, com o antebraço flectido aproximadamente a $90^{\circ} \mathrm{e}$ em supinação, com o punho fechado e colocado sobre a crista ilíaca. Deste posicionamento inicial, o membro superior direito efectua o choku-zuki com o máximo de velocidade e força até ao momento de contacto do punho com o alvo (Figura 1).

Cada sujeito realizou vinte repetições do choku-zuki, repartidas por 4 séries de 5 repetições, intervaladas 5 min. entre cada série, e com um intervalo de $5 \mathrm{~s}$ entre cada repetição. Em duas das séries o choku-zuki foi realizado com impacto efectivo e nas outras duas séries o soco parava voluntariamente no instante do contacto com a makiwara. As séries com impacto (CI) e sem impacto (SI) foram realizadas alternadamente.

\section{3.- Instrumentos}

Foram utilizados quatro sensores electromagnéticos que se encontravam colocados sobre a primeira vértebra torácica, na porção externa e medial do braço, na face posterior do antebraço junto do punho e na makiwara, $20 \mathrm{~cm}$ abaixo da zona alvo. Os sensores estavam ligados a um transmissor de grande alcance com uma área de registo de aproximadamente 2,50 $\mathrm{m}$ de raio. $0 \mathrm{~s}$ dados cinemáticos foram recolhidos com uma frequência de amostragem de $100 \mathrm{~Hz}$ ("Flock of Birds2" System Ascension Technology, Software - Motion Monitor version 6.05). Após a calibração (Meskers, CG, et al., 1999), o sistema apresentou uma precisão de 0,3 $\mathrm{mm}$ para a posição e 0,15을 para a orientação.

Os sinais da electromiografia (EMG) foram captados através de eléctrodos bipolares de superfície ativa (1992-2002 National Instruments, Frankfurt, Alemanha), com uma impedância de entrada de $10 \mathrm{G}^{\prime} \Omega$, o ruído de $1 \mu \mathrm{V}$, com uma relação de rejeição de modo comum (CMRR) de 120 $\mathrm{db}$, e um ganho de 2500. Os eléctrodos foram fixados sobre superfícies de deteção com $\mathrm{Ag} / \mathrm{AgCl}$, descartáveis e autoadesivas (Medicoelectronics - Copenhagen, Denmark), com os centros separados de $20 \mathrm{~mm}$ de distância entre si. Após a preparação da pele, os eléctrodos foram colocados na região mediana do ventre dos músculos deltoid nas suas porções anterior (DA) e posterior (DP), na porção clavicular (PC) e esternal (PS) do músculo pectoralis major, no músculo infraespinatus (IF), no músculo bíceps brachii (BB), no músculo brachioradialis (BR), na porção lateral do músculo tríceps brachii (TB) e no músculo pronator teres (PT). Estes foram os músculos estudados através do eletromiograma. 0 eléctrodo terra foi colocado sobre a quinta vértebra lombar.

0 registo EMG foi efectuado em simultâneo com registo dos sinais cinemáticos através de um conversor de 12-bits A/D (DaqCard ${ }^{T M}$ - 700, Multifunction I/0 from National Instruments), com uma taxa de amostragem de $1600 \mathrm{~Hz}$. Aquisição foi realizada através do DasyLab 6.0 (Biovision). 0 processamento do sinal EMG foi realizado com software desenvolvido em MatLab ® (The Mathworks Inc, Natick Massachusetts, USA).

\section{4.- Processamento de dados}

\section{Cinemáticos}

Os participantes foram previamente calibrados para o campo eletromagnético criado pela Flock of Birds2 (FOB), definindo-se o sistema de coordenadas locais do braço e antebraço em 
relação ao sistema global de coordenadas. Neste processo, os participantes foram posicionados de acordo com a posição anatómica descritiva, mas com o antebraço em semipronação. Esta posição define os planos corporais (sagital, frontal e transversal) e os seus respectivos os eixos de rotação (lateral, ântero-posterior e longitudinal ou vertical).

Este procedimento define os zeros posicionais dos segmentos, e serve de procedimento de normalização do sujeito, o que permite a posterior comparação entre os sujeitos. 0 centro de rotação na articulação glenoumeral foi determinado usando uma estimativa cinemática baseada no cálculo instantâneo dos eixos helicoidais, e uma estimativa geométrica com base no ajuste de uma esfera à superfície da cavidade glenoide (Meskers et al. , 1998; Veeger 2000).

Foi aplicada a dupla abordagem dos ângulos de Euler para maximizar a precisão sobre a computação do movimento tridimensional do ombro em relação aos eixos do movimento do sistema de coordenadas segmentares (Ying et al., 2002).

Os sinais cinemáticos recolhidos pelo FOB dos movimentos de flexão e rotação interna do braço na articulação do ombro, de extensão e de pronação do antebraço na articulação do cotovelo foram processados em MatLab. Foi aplicado aos dados cinemáticos um filtro passa-baixo Butterworth de $10 \mathrm{~Hz}$ de segunda ordem.

As variáveis dependentes cinemáticas foram: tempo de início e fim do movimento identificados a partir das curvas de movimento, a duração dos movimentos medidos entre o instante de início e o fim dos respetivos movimentos (tendo em consideração que a análise foi realizada apenas até ao instante do contacto com o alvo), ângulo posicional dos segmentos em estudo, a velocidade e aceleração angular e respetivos picos, calculadas pelo software Motion Monitor version 6.05 por derivação (1 $1^{\underline{a}}$ e $2^{\underline{a}}$ derivada) das curvas de posição (Figura 2).

A identificação e marcação dos instantes de início e fim dos movimentos foi efectuada visualmente nos gráficos gerados pelo MatLab, por um único investigador treinado para este fim, e registados automaticamente, dando continuidade ao restante processamento dos dados.

Figura 2

Ilustração representativa das curvas de posição referentes aos dados cinemáticos do braço e antebraço na execução do choku-zuki
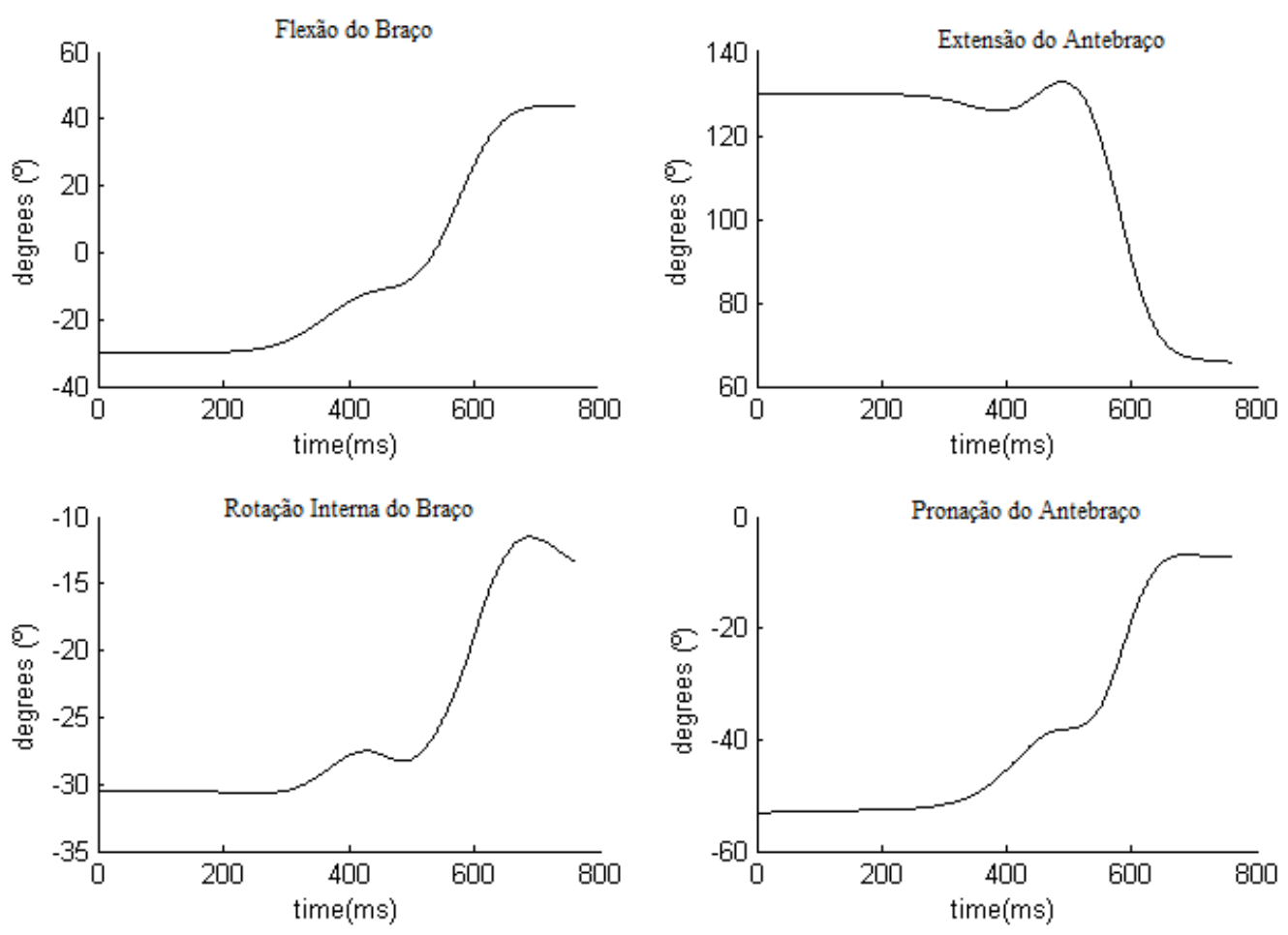
$E M G$

Os sinais EMG dos músculos estudados foram digitalmente filtrados (10 a $400 \mathrm{~Hz}$ ), rectificados e suavizados com um filtro passa-baixa de $50 \mathrm{~Hz}$ (Butterworth, $4^{\mathrm{a}}$ ordem). Sinais EMG foram normalizados tendo como referencia os sinais EMG da contracção voluntária máxima isométrica (CVMI). Para este procedimento foram realizadas três CVMI para cada músculo isolado, com 1 minuto de descanso entre cada CVMI. Os dados foram recolhidos com os segmentos posicionados de forma a que cada músculo tivesse uma intervenção preferencial como agonista para desenvolver a sua intensidade máxima de activação. Na definição posicional dos segmentos para este momento foram seguidas as recomendações de Basmajian \& DeLuca (1985).

Na execução do choku-zuki, as curvas de EMG de cada uma das execuções foram isolados e recolhidos os parâmetros de tempo e amplitude. Nos músculos BB, BR e PT evidenciou-se a existência de duas ativações diferentes. Estes dois momentos distintos de activação estão referenciados como primeiro (BB1, BR1, PT1) e segundo períodos (BB2, BR2, PT2) de activação.

No EMG normalizado, rectificado e suavizado, as variáveis dependentes estudadas foram o instante de início (onset) e fim (offset) da activação dos músculos, a intensidade da actividade muscular através da raiz quadrada média da activação muscular (RMS) em cada um dos músculos estudados, e o instante do pico de actividade muscular (Figura 3).

\section{Figura 3}

Ilustração representativa das curvas de EMG dos vários músculos estudados na execução do choku-zuki. Pectoralis major porção clavicular (PC) e esternal (PS), infraespinatus (IF), deltoid anterior (DA) e posterior $(D P)$, tríceps brachii (TB), bíceps brachii (BB), pronator teres (PT) brachioradialis (BR)

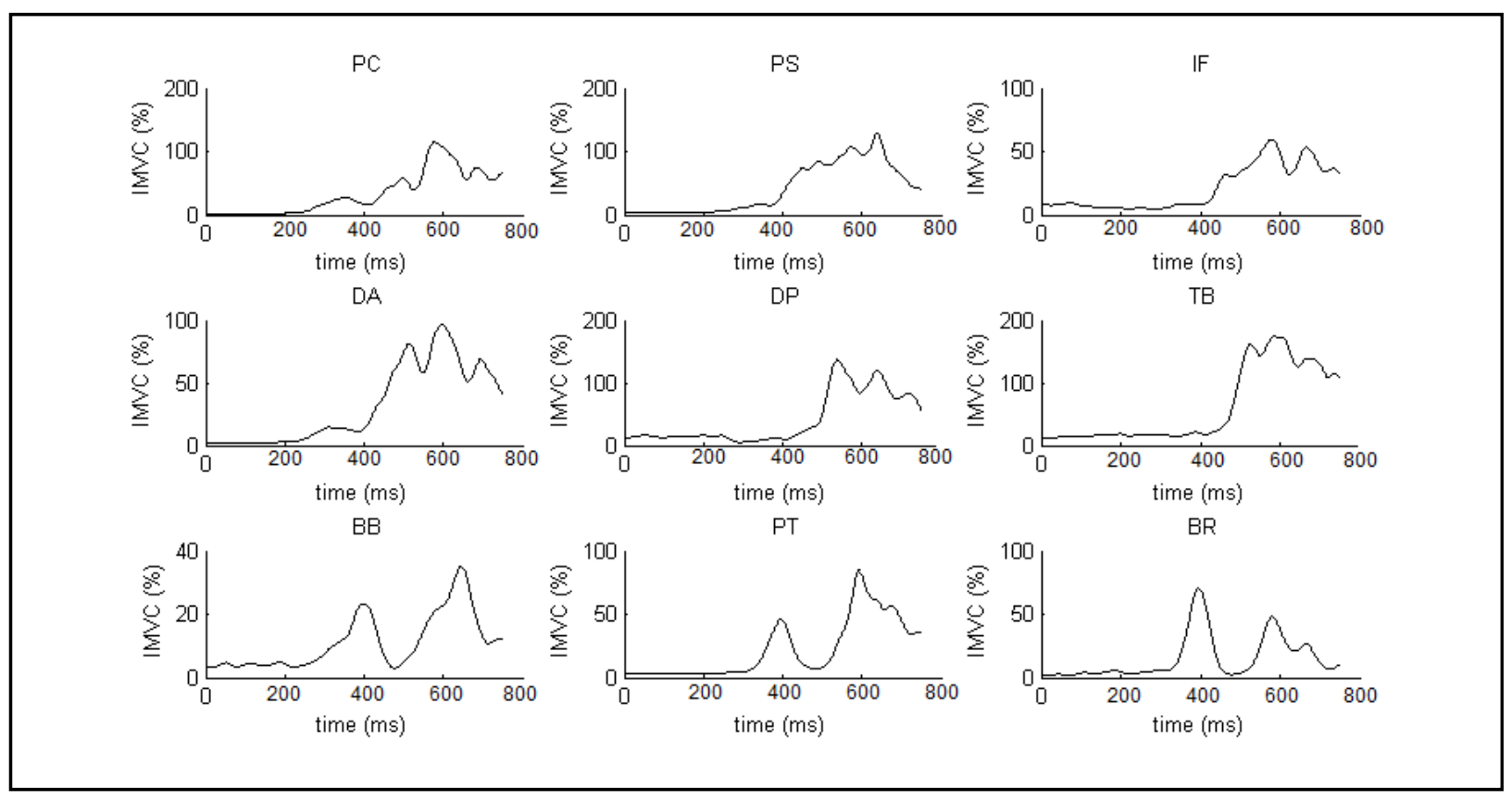

A determinação do onset/offset de ativação dos músculos foi feita a partir da identificação de um valor médio de actividade, calculado sobre um intervalo de tempo de $250 \mathrm{~ms}$, no início do registo do EMG. A actividade média calculada mais 3 vezes o desvio-padrão foi considerada como o limite de intensidade que uma vez atingido e mantido por $25 \mathrm{~ms}$ identificou o tempo de onset/offset da ativação EMG (Hodges \& Bui, 1996; Giakas \& Baltzopoulos, 1997; Micera et al. , 1998; Roetenberg et al. , 2003).

Os EMG e os dados cinemáticos foram sincronizados por um sinal de disparo simultaneamente registrado nos ficheiros de dados cinemáticos e de EMG. Também foram sincronizados através do sinal do sensor FOB colocado na makiwara e que identificou o tempo de contacto, que correspondia ao tempo final de análise. 


\section{5.- Estatística}

Os dados descritivos encontram-se representados pelos valores médios e respectivos desvio padrão.

A comparação de variáveis dependentes cinemáticas e electromiográficas entre as execuções com impacto e sem impacto foi efectuada com a utilização do Independent-Samples T Test. Foi verificada a normalidade da amostra (Kolmogorov-Smirnov test). Neste tratamento foi considerado um nível de significância de $\mathrm{p} \leq 0.05$. A análise estatística foi efectuada com o SPSS para o Windows.

\section{3.- Resultados}

\section{1.- Descrição cinemática e comparação}

A Tabela 2 apresenta as variáveis dependentes cinemáticas nas execuções do choku-zuki com impacto e sem impacto, assim como as diferenças significativas.

Tabela 2

Média e desvio padrão das variáveis cinemáticas na execução do choku-zuki com impacto (CI) e sem impacto (SI)

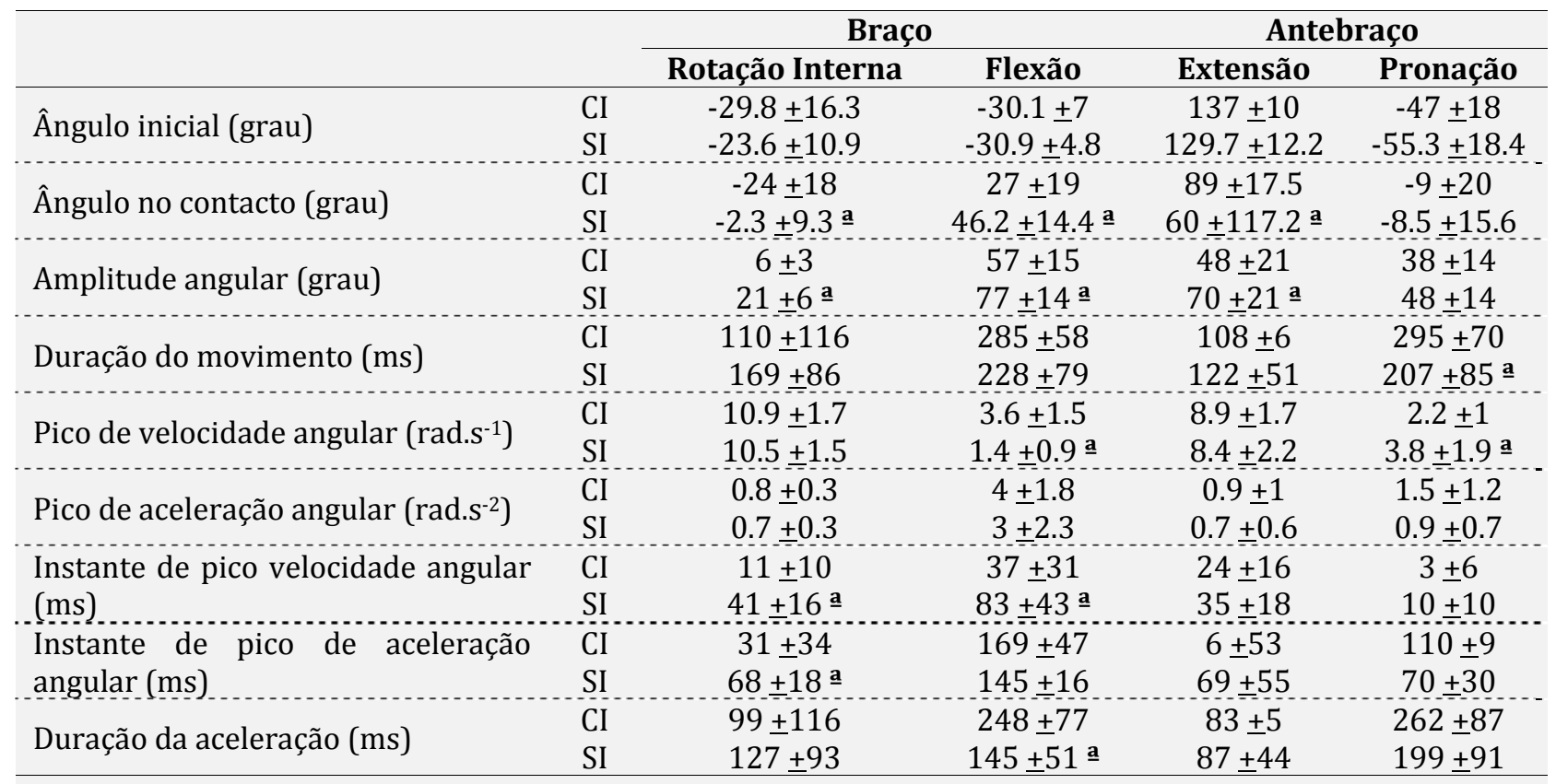

a Indica a existência de diferenças estatísticas entre condição de execução $(p \leq .05)$.

De acordo com a tabela 2, a sequência cinemática dos movimentos segmentares na execução do choku-zuki com impacto, começa através da pronação do antebraço no cotovelo seguida da flexão do braço na articulação do ombro aos $295 \mathrm{~ms}$ e $285 \mathrm{~ms}$ antes do contato, respetivamente, seguindo-se a rotação interna do braço na articulação do ombro e a extensão do antebraço no cotovelo que se iniciam aos $110 \mathrm{~ms}$ e $108 \mathrm{~ms}$ antes do contato, respetivamente.

A flexão do braço no ombro inicia-se a partir de uma extensão de $-30^{\circ}$ e e efetua um movimento de amplitude média de 57우 ; sendo acelerado durante de $248 \mathrm{~ms}$ e é o primeiro movimento a atingir o pico máximo de velocidade angular, aos $37 \mathrm{~ms}$ antes do contato. A pronação do antebraço no cotovelo é realizada com uma amplitude de $38^{\circ}$ a partir do posicionamento inicial em supinação, sendo este movimento acelerado durante $262 \mathrm{~ms}$, atingindo o pico de velocidade angular $3 \mathrm{~ms}$ antes do contato. A rotação interna do braço no ombro inicia-se a partir de uma posição em rotação externa de $-30^{\circ}$ e efectuando uma rotação de amplitude média de cerca de $6^{\circ}$, estando o movimento em aceleração durante $99 \mathrm{~ms}$, com o pico de velocidade angular a acontecer $11 \mathrm{~ms}$ antes do contato. 0 movimento final de condução do punho em direção á makiwara, a 
extensão do antebraço no cotovelo, inicia-se a partir de um posicionamento em flexão de 137으, realizando um movimento de extensão de $48^{\circ}$ de amplitude e atingindo o pico de velocidade angular $24 \mathrm{~ms}$ antes do contato.

Quando comparadas as execuções do choku-zuki com impacto e sem impacto verifica-se que existem diferenças significativas $(\mathrm{p}<0,05)$ em diversas variáveis nos diferentes movimentos segmentares. Especificamente, na duração do movimento, que reflecte a diferença entre o tempo de início e o contato, onde na execução com impacto a pronação do antebraço no cotovelo começa mais cedo e demora mais tempo do que na execução sem impacto. Por outro lado, a amplitude angular dos diferentes movimentos segmentares é significativamente menor na execução com impacto, com excepção do movimento de pronação, o que reflecte as diferenças significativas encontradas no posicionamento angular dos segmentos no instante do contato.

Verifica-se também que na execução do choku-zuki com impacto o instante em que é atingido o pico de velocidade angular dos movimentos do braço acontece mais perto do final da execução do que quando este é voluntariamente parado, não efectuando impacto. Nos movimentos do antebraço também se verifica existir uma tendência semelhante mas sem ser significativamente diferente. Todavia, apenas no movimento de rotação interna do braço no ombro se verifica existir diferenças significativas no instante de ocorrência do pico de aceleração mostrando que este acontece mais perto do contato na execução com impacto.

\section{2.- Descrição EMG e comparação}

A Tabela 3 apresenta as variáveis EMG dependentes e os resultados relativos às execuções com impacto e sem impacto, com a indicação das diferenças significativas entre execuções.

Tabela 3

Média e desvio padrão das variáveis electromiográficas dos músculos na execução do choku-zuki com impacto (CI) e sem impacto (SI)

\begin{tabular}{|c|c|c|c|c|c|c|c|}
\hline & & PC & PS & IF & DA & DP & TB \\
\hline \multirow{2}{*}{$\begin{array}{l}\text { Onset ativação muscular } \\
\text { (ms) }\end{array}$} & CI & $285 \pm 48$ & $237 \pm 2$ & $150 \pm 32$ & $262 \pm 52$ & $141 \pm 54$ & $143 \pm 34$ \\
\hline & SI & $256+5$ & $245+24$ & $160+37$ & $256+44$ & $139+57$ & $151+25$ \\
\hline \multirow{2}{*}{$\begin{array}{l}\text { Offset ativação muscular } \\
\text { (ms) }\end{array}$} & CI & 0 & 0 & 0 & 0 & 0 & 0 \\
\hline & SI & 0 & $1+4 \underline{\mathbf{a}}$ & 0 & 0 & 0 & 0 \\
\hline \multirow{2}{*}{ Instante do pico EMG (ms) } & CI & $97 \pm 25$ & $87 \pm 22$ & $36 \pm 29$ & $82 \pm 36$ & $18 \pm 14$ & $33 \pm 25$ \\
\hline & SI & $95+36$ & $101+37$ & $4+27 \underline{a}$ & $92+35$ & $28 \pm 15$ & $44 \pm 23$ \\
\hline \multirow{3}{*}{ Intensidade (RMS-\% CVMI) } & $\mathrm{CI}$ & $104 \pm 0.7$ & $98 \pm 0.4$ & $71 \pm 0.2$ & $82 \pm 0.2$ & $111 \pm 0.6$ & $97 \pm 0.3$ \\
\hline & SI & $171 \pm 1.2$ & $89+0.4$ & $66+0.2$ & $75+0.3$ & $122+0.6$ & $102+0.4$ \\
\hline & & BB1 & BB2 & PT1 & PT2 & BR1 & BR2 \\
\hline \multirow{2}{*}{$\begin{array}{l}\text { Onset ativação muscular } \\
\text { (ms) }\end{array}$} & CI & $250 \pm 27$ & $76 \pm 4$ & $237 \pm 43$ & $83 \pm 4$ & $234 \pm 34$ & $76 \pm 23$ \\
\hline & SI & $255+2$ & $64+19 \underline{\mathbf{a}}$ & $234+33$ & $69+11 \mathrm{a}$ & $246+24$ & $79+29$ \\
\hline \multirow{2}{*}{$\begin{array}{l}\text { Offset ativação muscular } \\
\text { (ms) }\end{array}$} & CI & $124 \pm 125$ & 0 & $11 \pm 25$ & 0 & $12 \pm 23$ & 0 \\
\hline & SI & $130+25$ & 0 & $10 \overline{6}+9$ & 0 & $135+29$ & 0 \\
\hline \multirow{2}{*}{ Instante do pico EMG (ms) } & CI & $192 \pm 24$ & $26 \pm 3$ & $192 \pm 39$ & $13 \pm 0.19$ & $188 \pm 33$ & $16 \pm 16$ \\
\hline & SI & $196+9 \mathbf{a}$ & $16+18 \mathbf{a}$ & $179+28$ & $18+9 \underline{\mathbf{a}}$ & $198+27$ & $22+17$ \\
\hline \multirow{2}{*}{ Intensidade (RMS-\% CVMI) } & $\mathrm{CI}$ & $32 \pm 0.2$ & $19 \pm 0.2$ & $45 \pm 0.2$ & $67 \pm 0.5$ & $50 \pm 0.3$ & $33 \pm 0.2$ \\
\hline & SI & $42 \pm 0.3$ & $30 \pm 0.2$ & $51 \pm 0.2$ & $85 \pm 0.5$ & $62 \pm 0.2$ & $38 \pm 0.2$ \\
\hline
\end{tabular}

PC - porção clavicular do pectoralis major; PS - porção esternal do pectoralis major; IF - infraespinatus; DA porção anterior do deltoid; DP - porção posterior do deltoid; TB - porção lateral do triceps brachii; BB - biceps brachii; PT - pronator teres; BR - brachioradialis.

a Indica a existência de diferenças estatísticas entre condição de execução $(p \leq .05)$.

A sequência encontrada relativa ao instante de ativação dos músculos na execução do choku-zuki com impacto mostra que os músculos que primeiro a são ativados são os agonistas da flexão e rotação interna do braço no ombro (PC, DA, PS), entre os $285-237$ ms antes do contato. A estes segue-se a ativação inicial dos músculos flexores e pronador do antebraço na articulação do cotovelo (BB1, PT1, BR1), entre os 250-234ms. No cotovelo o músculo extensor do antebraço (TB) 
inicia a sua atividade um pouco mais tarde, aos $143 \mathrm{~ms}$, seguido do segundo instante de ativação do músculo pronador do antebraço (PT2).

A atividade dos músculos antagonistas, da rotação interna e flexão do braço na articulação do ombro (IF, DP) começa aos 150ms e 141ms antes do contato com a makiwara, respetivamente, e aproximadamente $100 \mathrm{~ms}$ depois da ativação dos músculos agonista. Os músculos antagonistas da extensão e pronação do antebraço no cotovelo (BB2, BR2) são os últimos a ser ativados.

Em relação ao pico de atividade dos músculos da flexão e da rotação interna do braço no ombro (PC, DA, PS), este acontece entre os $97-82 \mathrm{~ms}$ antes do contato. Já nos músculos antagonistas destes movimentos (IF, DP) este pico acontece mais perto do instante de contato (36ms e 18ms). Relativamente à intensidade de ativação dos músculos nesses movimentos, os músculos agonistas (PC, DA, PS) apresentam um RMS entre 82 e 100\% do CVMI, e os antagonistas apresentam um RMS perto dos 71\% do CVMI no músculo IF e um RMS perto dos 111\% do CVMI no músculo DP.

Relativamente aos músculos responsáveis pela extensão e pronação do antebraço no cotovelo, o TB é claramente o que tem maior intensidade de ativação, perto de $100 \%$ da CVMI. Contudo, verifica-se que o músculo PT apresenta maior intensidade de atividade na sua segunda ativação. Nos músculos antagonistas (BB, BR) a intensidade de atividade é igual ou inferior a 50\% do RMS da CVMI em qualquer dos períodos de activação.

Comparando a solicitação e atividade muscular entre as duas execuções do choku-zuki, a variável EMG com maior variação entre execuções é o instante de ocorrência do pico de atividade, onde na execução com impacto existe um intervalo significativamente maior de tempo entre o instante do pico de atividade e o contato no músculo IF, relativo ao braço na articulação do ombro e no músculo BB2 relativo ao antebraço na articulação do cotovelo. Todavia o intervalo de tempo entre o instante do pico de atividade e o contato é significativamente menor nos músculos BB1 e PT2 do antebraço no cotovelo.

Relativamente ao instante de ativação muscular verifica-se que o músculo BB2, antagonista da flexão do antebraço no cotovelo, e o músculo PT2, agonista da pronação do antebraço no cotovelo, são ativados significativamente mais cedo na execução com impacto. Nos restantes músculos não existe qualquer diferença significativa entre execuções nesta variável.

Relativamente à intensidade da atividade muscular medida através do RMS, não existem diferenças significativas entre a execução do choku-zuki com impacto e sem impacto na makiwara.

\section{4.- Discussão}

\section{1.- Descrição do choku-zuki}

De acordo com o objetivo deste trabalho, de caracterizar a técnica de socar choku-zuki quando executada com impacto por karatecas experientes, os resultados obtidos demonstram que estes usam um padrão de movimento que tem uma sequência de desenvolvimento segmentar no sentido próximo - distal mas com a antecipação do início do movimento de pronação do antebraço no cotovelo, e com os movimentos do braço na articulação do ombro a atingirem o pico de velocidade angular tendencialmente antes dos movimentos do antebraço.

0 pico de velocidade angular dos movimentos realizados em torno do eixo longitudinal (rotação interna do braço no ombro e pronação do antebraço no cotovelo) ocorre depois dos movimentos executados em torno do eixo lateral (flexão do braço no ombro e extensão do antebraço no cotovelo). Por outro lado, a extensão do antebraço no cotovelo atinge um maior pico de velocidade angular que a flexão de braço no ombro, o que sugere a existência de transferência de velocidade do segmento proximal para o distal durante o movimento (Van Gheluwe \& Hebbelink, 1985; Putnam, 1993; Hirashima et al. , 2002). Esse transfer poderá resultar num aumento de velocidade e potencia na execução do choku-zuki. Todavia, este comportamento não é observado na rotação internado braço no ombro relativamente à pronação do antebraço no cotovelo.

0 pico de velocidade angular dos movimentos segmentares encontra correspondência na sequência de ocorrência do instante de pico da actividade muscular. Verifica-se que a ativação 
muscular começa através da ativação dos músculos da flexão e rotadores internos do braço, atingindo estes o seu pico de atividade antes do contato e antes do pico de velocidade angular, respectivamente, da flexão do braço e rotação interna na articulação do ombro.

Os músculos antagonistas dos movimentos do braço na articulação do ombro e o principal responsável pela extensão do antebraço na articulação do cotovelo têm o seu pico de atividade a acontecer mais perto do instante de contato. Finalmente, nos últimos ms que precedem o instante de contato, o músculo da pronação e os músculos antagonistas à extensão do antebraço na articulação do cotovelo são novamente ativados, com seu pico de atividade a surgir próximo do instante de contato.

O primeiro período de ativação dos músculos flexores (BB1, BR1) e pronadores do antebraço (PT1) tem como função a estabilização do cotovelo, preparando o antebraço para a aceleração que se segue. Estes resultados são apoiados por Neto e Magini (2008) que mostraram que BB assiste durante a flexão do braço no ombro, e segundo McGill et al. (2010) os dois períodos de ativação do BB, BR e PT também são comuns na atividade muscular de lutadores de artes marciais mistas, o que aumenta o controlo dos movimentos segmentares e a eficácia do soco.

No que diz respeito à atividade dos músculos e aos processos de coordenação entre músculos agonistas e antagonistas, parece evidenciar-se a existência de um padrão fásico da inervação recíproca, semelhante ao que acontece com outros movimentos de características balísticas (Desmedt \& Godaux, 1979; Wadman et al. , 1979; Le Bozec et al., 1980), mas em que no choku-zuki com impacto o período de co contração tende a ser superior a $100 \mathrm{~ms}$ nos músculos do braço e 70 ms nos músculos do antebraço, o que são valores superiores aos que são referidos nesses estudos. Todavia, este maior período de co contração muscular deverá estar relacionado com a necessidade de socar com precisão a makiwara.

Da análise do valor normalizado do RMS, surpreendentemente, o músculo que apresenta maior intensidade de ativação é o DP, revelando a importância da travagem do movimento de flexão do braço na articulação do ombro, onde esta ação poderá funcionar como um mecanismo de proteção da articulação (Wilk et al., 1997), mas também transferir aceleração e velocidade para o segmento distal, em consequência da desaceleração do segmento proximal, o que poderá incrementar a potência final do choku-zuki.

Este incremento da ativação em músculos antagonistas foi anteriormente identificado por Sbriccoli et al. (2010) em karatecas experientes ao verificar que estes possuem um alto nível de ativação dos músculos antagonista durante a execução de um pontapé frontal.

A maior intensidade de activação dos músculos agonistas da flexão e rotação interna do braço na articulação do ombro realça a sua importância na aceleração do segmento, bem como a transferência de energia para a extensão do antebraço na articulação do cotovelo, onde o TB tem também uma grande intensidade de ativação. Witte et al. (2005) encontraram também no músculo TB uma grande intensidade de activação durante a execução de um de soco de karaté, assim como Neto e Magini (2008) na execução de um ataque de mão aberta de Kung Fu. Além disso, Dinn e Behm (2007) verificaram existir um aumento significativo de ativação nos músculos pectoralis major e TB, após treino dinâmico e isométrico do movimento de socar.

O músculo IF, além de ser antagonista da rotação interna do braço no ombro, encontra-se envolvido na estabilidade dinâmica da articulação do ombro, o que é importante nos movimentos de socar. 0 músculo pronador do antebraço na articulação do cotovelo apresenta uma ativação menos intensa, mas maior do que a que se verifica nos músculos flexores do antebraço, estando estes apenas envolvidos no controlo dos movimentos do antebraço e sua estabilização na articulação do cotovelo.

\section{2.- Comparação entre a execução CI e SI}

É assumido que o treino, nomeadamente de karaté, conduz à aprendizagem e alteração das estratégias de controlo motor aumentando assim a performance motora. A prática de tarefas em acções motoras apropriadas, mas que de alguma forma podem ser diferentes, conduz à aquisição, 
melhoria e estabilização dos padrões de coordenação neuromuscular que se podem traduzir em diferenças cinesiológicas no movimento. Assim, era nossa expectativa desde o início deste trabalho que quando comparada a execução do choku-zuki com impacto com a execução sem impacto, as componentes cinesiológicas do movimento apresentassem diferenças significativas nos diferentes parâmetros, permitindo identificar o que distinguia as duas execuções entre si quando efetuadas por karatekas experientes.

Relativamente à literatura disponível esta identifica que com o treino há uma melhor ativação neuromuscular na realização de habilidades motoras complexas, o que é associado no caso do soco com o aumento da velocidade do membro superior e do impulso (Cesari \& Bertucco, 2008). No presente trabalho a complexidade é modificada pela relação entre o impacto efetivo e a não existência de impacto. Contudo, na comparação entre atletas de karaté experientes com inexperientes em movimentos técnicos executados com o joelho foi verificado um aumento do "torque" isocinético, o que sugere uma melhoria no recrutamento de unidades motoras (Sbriccoli et al., 2010). Já no kung fu, os profissionais experientes apresentam menor atividade muscular antagonista do que participantes inexperientes na realização da técnica de socar com a palma da mão (Neto \& Magini, 2008).

Todavia, aquilo que vulgarmente acontece em treino no Dojo, o ensino técnico sem contacto como fator facilitador de aprendizagem e aperfeiçoamento, e posterior execução da mesma técnica com contacto e impacto num alvo não tem merecido a atenção e reflexão da comunidade científica dos desportos de combate, não sendo possível analisar os resultados encontrados em confronto com outros trabalhos produzidos.

No presente estudo, a execução do choku-zuki com impacto começa mais cedo, e atinge o pico de velocidade angular dos movimentos do braço realizados na articulação do ombro e do antebraço realizados na articulação do cotovelo mais perto do instante de contacto, o que mostra que a travagem dos movimentos segmentares acontece mais tarde, conduzindo a um impacto efectivo. Contudo, apresenta uma sequência próximo-distal semelhante à da execução sem impacto nos movimentos feitos no plano sagital.

Os movimentos produzidos em torno do eixo longitudinal na execução com impacto obedecem a uma lógica diferente da que se verifica na execução sem impacto, e também da que é descrita empiricamente pelos mestres de karaté. A antecipação significativa do instante do pico de velocidade angular do movimento de pronação do antebraço na execução sem impacto, provavelmente relacionada com o menor valor do pico de aceleração angular neste tipo de execução, permite estabilizar e posicionar mais cedo o punho relativamente ao instante de impacto, e parar o movimento antes do impacto efectivo.

Todavia, o menor pico de velocidade angular do movimento de pronação do antebraço no cotovelo na execução com impacto, a antecipação do início deste movimento e a menor amplitude evidenciam uma maior preocupação com a necessidade de proteção das estruturas anatómicas no contato efetivo com o makiwara, nomeadamente o punho. Esta ação protetora poderá ter como consequência uma diminuição do impacto no alvo, para além de que a antecipação do movimento de pronação poderá ser um indicador/estímulo perceptível pelo oponente de que o ataque se vai iniciar, o que lhe permitirá antecipar a sua resposta.

Na verdade, é a flexão do braço no ombro que mais diferencia as execuções com impacto das sem impacto. Além do maior valor da velocidade angular e duração da aceleração na execução com impacto, com o pico de velocidade angular a acontecer mais perto do instante de contacto do que na execução sem impacto, com uma menor amplitude de movimento mas com maior duração, e com menor grau de flexão no instante do contato. Alterações estas que reflectem a aprendizagem ao contacto efectivo, permitindo uma protecção das estruturas articuladas, mas possibilitando o transfer energético para o segmento mais distal, importante para potenciar o impacto no alvo.

A menor amplitude dos movimentos segmentares na execução com impacto estão de acordo com a tendência referida na literatura relativa a alterações nos padrões cinemáticos que são modificados durante a aprendizagem das diferentes tarefas motoras (Hobart et al. , 1978; Jaegers et al. , 1989; Pezarat-Correia et al. , 2001). Contudo precisamos de considerar que a execução sem impacto é o primeiro patamar de aprendizagem ao qual se sucede a execução com impacto. 
Relativamente à atividade muscular os karatecas experientes ao apresentam o pico de atividade a acontecer mais perto do instante de contato nos músculos agonistas do braço e antebraço na execução do choku-zuki com impacto, identifica-se a existência efetiva de uma transferência de força muscular contráctil máxima a acontecer muito perto do contacto com a makiwara, o que resultará num aumento da potência no instante do impacto no alvo.

Esta acção potenciadora do impacto parece ser ajudada pelo surgimento mais tardio do pico de actividade do músculo antagonista BB1 relativo à sua acção no movimento de extensão do antebraço no cotovelo e a sua estabilização, mas também pela antecipação do pico de activação do músculo IF, que tenderá a estabilizar mais cedo o movimento de rotação interna do braço no ombro. Este conjunto de acções musculares são relevantes na estabilização segmentar no impacto efetivo, podendo aumentar a potência do soco e a proteção dos conjuntos articulados do membro superior.

Como foi exposto, apenas foram encontradas diferenças nas estruturas temporais da ativação EMG entre as execuções, mantendo-se semelhante a intensidade de ativação dos músculos estudados. Estas diferenças relativas aos tempos no EMG são a razão das alterações cinemáticas entre as execuções e responsáveis pela diferente performance na execução do choku-zuki na ação com impacto, o que é também defendido por Liang et al. (2008) que diz que um melhor desempenho balístico se deve principalmente às modulações temporais da actividade muscular agonista e antagonista.

\section{5.- Conclusões}

A execução do choku-zuki com impacto evidencia a existência de um melhor desempenho balístico identificado pelo pico de velocidade angular e pico de atividade muscular que acontecem mais próximo do instante do contato com a makiwara, verificando-se também uma tendência a uma menor amplitude do movimento e alterações na sua duração. Todavia, verifica-se que contrariamente ao que é empiricamente definido pelos mestres e ensinado no treino, como sendo a sequência correta de execução do choku-zuki, o movimento de pronação do antebraço tende a ser iniciado muito cedo pelos karatecas experientes, em qualquer das variantes de execução, com impacto ou sem impacto. Esta antecipação parece resultar da necessidade de posicionar corretamente o punho para realizar o impacto no alvo sem que desse impacto resulte dano/lesão nas estruturas corporais que contactam o alvo.

Com os resultados encontrados e expostos, torna-se evidente que é muito importante a aprendizagem e treino das técnicas de socar realizadas com impacto efetivo. Esse treino promove aprendizagens motoras e adaptações cinemáticas e neuromusculares no praticante que conduzem há realização do soco com uma eficácia maior e em maior segurança. Provavelmente o incremento do tempo de treino técnico realizado com impacto trará maior segurança ao praticante na execução das técnicas e tenderá a reduzir a antecipação do movimento de pronação do antebraço na articulação do cotovelo, o que também tenderá a diminuir o tempo de execução do soco.

\section{Referências}

Basmajian, J.V., \& De Luca, C.J. (1985). Muscles alive. Their functions revealed by electromyography. Baltimore: William \& Wilkins.

Bledsoe, G.H., Hsu, E.B., Grabowski, J.G., Brill, J.D., \& Li, G. (2006). Incidence of injury in professional mixed martial arts competitions. Sports Science and Medicine, CSSI, 136-142.

Cesari, P., \& Bertucco, M. (2008). Coupling between punch efficacy and body stability for elite karaté. J Sci Med Sport, 11(3), 353-356.

Courtonne, C. (1996). Physique et Karaté. Le secret de la puissance. Paris: Editions Chiron.

Desmedt, J.E., \& Godaux, E. (1979). Voluntary motor commands in human ballistic movements. Ann Neurol, 5(5), 415-421.

Dinn, N.A., \& Behm, D.G. (2007). A comparison of ballistic-movement and ballistic-intent training on muscle strength and activation. Int J Sports Physiol Perform, 2(4), 386-399. 
Giakas, G., \& Baltzopoulos, V. (1997). A comparison of automatic filtering techniques applied to biomechanical walking data. J Biomech, 30(8), 847-850.

Hallett, M., Shahani, B.T., \& Young, R.R. (1975). EMG analysis of stereotyped voluntary movements in man. J Neurol Neurosurg Psychiatry, 38(12), 1154-1162.

Hirashima, M., Kadota, H., Sakurai, S., Kudo, K., \& Ohtsuki, T. (2002). Sequential muscle activity and its functional role in the upper extremity and trunk during overarm throwing. J Sports Sci, 20(4), 301-310.

Hobart, D.J., Vorro, J.R., \& Dotson, C.O. (1978). Synchronized myoelectric and cinematographic analysis of skill acquisition. J Human Mov Stud, 4, 155-66.

Hodges, P.W., \& Bui, B.H. (1996). A comparison of computer-based methods for determination of onset of muscle contraction using electromyography. Electroencephalogr Clin Neurophysiol, 101(6), 511-519.

Jaegers, S., Peterson, R., Dantuma, R., Hillen, H., Geuze, R., \& Schellekens, J. (1989). Kinesiologic aspects of motor learning in dart throwing. J Hum Mov Stud, 16, 161-171.

Le Bozec, S., Maton, B., \& Cnockaert, J.C. (1980). The synergy of elbow extensor muscles during dynamic work in man: I. Elbow extension. Eur J Appl Physiol Occup Physiol, 44(3), 255-269.

Liang, N., Yamashita, T., Ni, Z., Takahashi, M., Murakami, T., Yahagi, S., \& Kasai, T. (2008). Temporal modulation of agonist and antagonist muscle activities accompanying improved performance of ballistic movements. Hum Mov Sci, 27(1), 12-28.

Link, N., \& Chou, L. (2011). The anatomy of martial arts, an illustrated guide to the muscles used inkey kicks, strikes \& throws. Ulysses Press.

McGill, S.M., Chaimberg, J.D., Frost, D.M., \& Fenwick, C.M. (2010). Evidence of a double peak in muscle activation to enhance strike speed and force: an example with elite mixed martial arts fighters. J Strength Cond Res, 24(2), 348-357.

Meskers, C.G.M., Helm, F.C.Tvd., Rozendaal, L.A., \& Rozing, P.M. (1998). In vivo estimation of glenohumeral joint rotation center from scapular bony landmarks by linear regression. $J$ Biomech, 31, 93-96.

Meskers, C.G.M., Fraterman, H., Helm, F.C.Tvd., Vermeulen, H.M., \& Rozing, P.M. (1999). Calibration of the "Flock of Birds" electromagnetic tracking device and its application in shoulder motion studies. J Biomech, 32(6), 629-633.

Micera, S., Sabatini, A.M., \& Dario, P. (1998). An algorithm for detecting contraction by EMG signal processing. Med Eng Phys, 20(3), 211-215.

Nakayama, M. (1983). Dynamic Karaté: Instruction by the Master. Tokyo: Kodansha International LTD.

Neto, O.P., \& Magini, M. (2008). Electromiographic and kinematic characteristics of Kung Fu YauMan palm strike. J Electromyogr and Kinesiol, 18(6), 1047-1052.

Pezarat-Correia. P., Santos, P., Veloso, A., \& Cabri, J. (2001). Differences in the agonist/antagonist EMG pattern during a throwing task performed by experimented dart throwers and untrained subjects. Med Sci Sports Exerc, 33(5), S216.

Putnam, C.A. (1993). Sequential motions of body segments in striking and throwing skills: descriptions and explanations. J Biomech, 26(1), 125-135.

Roetenberg, D., Buurke, J.H., Veltink, P.H., Forner Cordero, A., \& Hermens, H.J. (2003). Surface electromyography analysis for variable gait. Gait Posture, 18(2), 109-117.

Sanes, J.N., \& Jennings, V.A. (1984). Centrally programmed patterns of muscle activity in voluntary motor behaviour of humans. Exp Brain Res, 54(1), 23-32.

Sbriccoli, P., Camomilla, V., Di Mario, A., Quinzi, F., Figura, F., \& Felici, F. (2010). Neuromuscular control adaptations in elite athletes: the case of top level karateka. Eur J Appl Physiol, 108(6), 1269-1280.

Schimdt, R. (1993). Apprentissage moteur et performance. Paris: Vigot.

Schmidt, R.A., \& Wrisberg, C.A. (2000). Motor learning and performance. A problem-based learning approach. 2nd ed. Champaign, IL: Human Kinetics.

Van Gheluwe, B., \& Hebbelink, M. (1985). The kinematics of the service movement in tennis: A three-dimensional cinematographical approach. In Winter, D., Norman, R., Wells, R., Hayes, K., \& Patla, A., editors. Biomechanics IX-B (pp. 521-526). Champaign: Human Kinetics.

Veeger, H.E.J. (2000). The position of the rotation center of glenohumeral joint. J Biomech, 33(12), $1711-1715$. 
Wadman, W., Denier van der Gon, J.J., Geuze, R.H., \& Mol, C.R. (1979). Control of fast goal-directed arm movements. J Hum Mov Stud, 5, 3-17.

Wilk, K.E., Arrigo, C.A., \& Andrews, J.R. (1997). Current concepts: the stabilizing structures of the glenohumeral joint. J Orthop Sports Phys Ther, 25(6), 364-379.

Witt,e K., Emmermacher, P., Hofmann, M., Schwab, K., \& Witte, H. (2005). Electromyographic researches of gyaku-zuki in karaté kumite. In Wang, Q., editor. Proceedings of XXIII International Symposium on Biomechanics in Sports (pp. 861-865). Beijing: The People Sport Press.

Ying, N., \& Kim, W. (2002). Use of dual Euler angles to quantify the three-dimensional joint motion and its application to the ankle joint complex. J Biomech, 35(12), 1647-1657.

Zetaruk, M.N., Violan, M.A., Zurakowski, D., \& Micheli, L.J. (2005). Karaté injuries in children and adolescents. Br. J. Sports Med, 39, 29-33

Zetaruk, M.N., Violan, M.A., Zurakowski, D., Micheli, L.J. (2000). Karaté injuries in children and adolescents. Accident Analysis and Prevention, 32, 421-425.

\section{Datos biográficos de los autores}

António M. VencesBrito é Doutor em Ciências da Motricidade, Mestre em Treino de Alto Rendimento. É docente na Escola Superior de Desporto de Rio Maior - Instituto Politécnico de Santarém (Portugal). É membro do European College of Sport Science, da International Martial Arts and Combat Sports Scientific Society e do Centro de Investigação em Desporto Saúde e Desenvolvimento Humano. É Praticante de karate Shotokan desde 1975 e é treinador e formador da Federação Nacional de Karate - Portugal e da Associação de Karate do Distrito de Setúbal. E-mail: abrito@esdrm.ipsantarem.pt 